\title{
Pseudomonas aeruginosa Recombinant Flagellin Induced Poly-Isotypic Humoral Immune Responses in the Balb/C Mice
}

\author{
Arezoo Shajiei ${ }^{1,2}$, Arezoo Rezaei Malal ${ }^{3}$, Ghorbanali Shahabi ${ }^{3}$, Ramin Farhoudi ${ }^{4}$, Sobhan \\ Faezi ${ }^{5}$, Majid Tebianian ${ }^{6}$, Nooshin Sohrabi ${ }^{7}$, Mehdi Mahdavi ${ }^{1,{ }^{*}}$ \\ ${ }^{1}$ Department of Virology, Pasteur Institute of Iran, Tehran, IR Iran \\ 2 Molecular Pathology Laboratory, Ghaem Medical Center, Mashhad University of Medical sciences, Mashhad, IR Iran \\ ${ }_{4}^{3}$ Department of Immunology, Cellular and Molecular Research Center, Shahrekord University, Shahrekord, IR Iran \\ 4 Department of Laboratory Animal Science, Pasteur Institute of Iran, Tehran, IR Iran \\ 5 Department of Laboratory Animal Science, Pasteur Institute of Iran, \\ 6 Department of Bacteriology, Pasteur Institute of Iran, Tehran, IR Iran \\ ${ }^{7}$ Department of Biology, Payam Noor University, Pardis, Tehran, IR Iran \\ ${ }^{*}$ Corresponding author: Mehdi Mahdavi, Departments of Virology, Pasteur Institute of Iran, Tehran, IR Iran. Tel: +98-2166496682, Fax:+98-2166496682, E-mail: M_Mahdavi@pasteur. \\ ac.ir, Mahdavivac@gmail.com.
}

Received: Jun 11, 2012; Revised: September 19, 2012; Accepted: October 24, 2012

\begin{abstract}
Background: Pseudomonas aeruginosa is an opportunistic pathogen that infects people with immunocompromised defenses like neutropenic, burned, hospitalized, and cystic fibrosis (CF) patients.

Objectives: The main aim of this study was to explore the possibility of the recombinant type A flagellin (r-fla-A) in combination of Montanide ISA 70 as a candidate vaccine to promote the humoral and cellular immune responses against r-fla-A.

Materials and Methods: Recombinant flagellin was prepared in Montanide ISA 70 adjuvant; Mice were divided into two groups one of six. The lymphocyte proliferation assay was performed with Brdu/ELISA and IL-4 and IFN- $\gamma$ cytokine level assay was carried out to determine the pattern of immune response (Th1 vs. Th2). Specific antibody responses were measured with an optimized in direct ELISA and finally different isotype-specific antibodies including IgG1, IgG2a, IgG2b, IgG3, and IgM was measured with ELISA.

Results: Immunized mice with adjuvanted flagellin showed a considerably increased lymphocyte proliferation compared with the control group $(\mathrm{P}=0.004)$. High level of IL-4 and IFN- $\gamma$ secretion was observed in immunized mice compared with the control group $(\mathrm{P}=0.003$ and $\mathrm{P}=0.006$, respectively) with Th1 profile. In addition to the strong antibody-mediated immune response, we found that immunization of mice with r-fla-A induces specific IgG1, IgG2a, IgG2b, IgG3 and IgM antibodies that indicates a statistically significant difference with the control group $(\mathrm{P}=0.003, \mathrm{P}=0.004, \mathrm{P}=0.004, \mathrm{P}=0.006$ and $\mathrm{P}=0.004$ respectively $)$.

Conclusions: Our results demonstrated that r-fla-A could induce cellular and humoral immune response as proper stimulant of polyisotypic humoral responses.
\end{abstract}

Keywords: Flagellin; Montanide; Poly-Isotypic Antibodies;

\section{Background}

Pseudomonas aeruginosa is an opportunistic pathogen that affects people with cystic fibrosis (CF), cancer, severe burns and immunosuppressed individuals with high morbidity and mortality and is responsible for ventilator-associated and hospital-acquired infections (1). The antibiotic treatment of $P$. aeruginosa infections is limited due to the extensive inherent and acquired antibiotic resistance (2). The emergence of pathogenic drug-resistant strains is rapidly increasing; therefore, development of an effective vaccination strategy is very important. Flagella are major structures of the pathogenic $P$. aeruginosa that contribute to virulence, stimulation of TLR5-depen- dent immune responses, and provide motility, the ability to evade from toxic substances, increase adhesion, etc. (3).

Flagellin monomer (FliC) is the major protein component of the flagellar filament andthe most effective immunologic effectors of immune system. There are two distinct serotype of flagellin; type A and B with molecular masses of about $45 \mathrm{kDa}$ and $55 \mathrm{kDa}$, respectively (4). Recent findings emphasize on the essential role of the flagellin monomers in detection of microbes by the host and in the induction of immune responses (3). Flagellin can cause the activation of the host inflammatory response via TLR5. The outstanding point is that TLR5-binding domain of flagellin is not exposed in the intact flagella (1). 
Hence, the flagellin is a good stimulator to promote TLR5 signaling than intact flagella. Bacterial flagellin has been studied as a useful antigen model and recently found to be a target of T cells (5).

Consequently, the immunogenicity of flagellin is basic element for variety of vaccines (6). At present, it is clear that the flagellin monomer may be a proper target as a vaccine component, particularly as a carrier protection or an immunologic adjuvant $(1,7)$. Despite the critical role of antibody immune responses, studies have shown that cell mediated immune responses have a clearly protective efficacy in P. aeruginosa infected patients $(8,9)$. Johansen and colleague reported that IFN- $\gamma$ treatment of $P$. aeruginosa infected rats was shown to reduce the chronic lung inflammation from an acute type dominated by neutrophils to an inflammation dominated by monomorphonuclear cells (10).

Adjuvant is necessary to promote strong adaptive responses using non-specific component of immune responses (11). As a result, there is an urgent need for development of new and improved vaccine adjuvants based on recombinant proteins. Therefore, it is important to develop non-live vaccine formulations capable of generating $\mathrm{T}$ helper 1 (Th1) responses in humans (12). There is a strong correlation between innate and adaptive immune responses, so strong innate immune response stimulation could induced strong adaptive immune responses (13). Recent studies have shown that the flagellin as a vaccine candidate or an immunologic adjuvant could improve the vaccine efficacy against $P$. aeruginosa infections $(14,15)$.

In the previously studies, we cloned and expressed fliC gene (type A flagellin-encoded gene) in Escherichia coli BL21 strain (DE3) as a bacterial expression system (16), and also found that passive therapy with anti-recombinant type A flagellin IgG would protect the burned mice (17). Our recent study demonstrated that active immunization with recombinant type A flagellin could protect burned mice against lethal $P$. aeruginosa infection by pathogen immobilization and promoting the phagocytic activity (unpublished result). Cytokines and antibody isotypes are important factors of the host immune system against $P$. aeruginos $a$ infections. TNF- $\alpha$ and IL- 4 were shown to enhance bacterial clearance, whereas IL-1 and IL-18 were defective in bacterial elimination (18). The role of other cytokines on resolution of infection is not wellunderstood.

\section{Objectives}

The main purpose of the present study was an explanation on the possibility of using flagellin in combination with Montanide ISA 70 as a candidate vaccine against $P$. aeruginosa and also to investigate whether flagellin would promote the humoral and cellular immune response. Here, for the first time we have shown that flagellin is able to induce poly-isotypic humoral immune response.

\section{Materials and Methods}

\subsection{Animals}

Eight to ten weeks old female Balb/C mice were obtained from the Pasteur Institute (Tehran, Iran). Mice were housed for one week before the experiment, given free access to food and water and maintained in a light/ dark cycle with lights ( $12 \mathrm{~h} / 12 \mathrm{~h})$. All experiments were in accordance with the Animal Care and Use Protocol of Pasteur Institute of Iran (13).

\subsection{Recombinant Flagellin Production and Purifi- cation}

In the previous study, recombinant type A flagellin gene was cloned and expressed (r-fla-A) as Histidine-tagged protein in a bacterial expression system (16). Briefly, P. aeruginosa type A flagellin gene (fliC) was first cloned into the expression vector (pET-28a) and then expressed in E.coli, BL-21(DE3) and finally purified by $\mathrm{Ni}^{++}$affinity chromatography.

\subsection{Vaccine Preparation}

Candidate vaccine was prepared in Montanide ISA 70 adjuvant (Seppic, France). to this purpose, r-fla-A protein was dissolved in PBS and mixed with Montanide ISA 70 with a 3:7 ratio v/v of. Mixture was prepared through stirring on a rotator at $3000 \mathrm{rpm}$ for $60 \mathrm{~min}$ until a stable white emulsion developed. Each dose comprises of $50 \mu \mathrm{L}$ of adjuvant-vaccine mixture containing $20 \mu \mathrm{g}$ of candidate vaccine (19).

\subsection{Experimental Groups and Immunization}

Mice were divided into two groups of six. The first group was immunized intradermally on days 0,14 and 21 with $50 \mu \mathrm{L}$ of candidate vaccine formulated in Montanide ISA 70 adjuvant with a dose of $20 \mu \mathrm{g}$. The second group was immunized intradermally on days 0,14 and 21 with $50 \mu \mathrm{L}$ of sterile PBS as the control group.

\subsection{Lymphocyte Proliferation Assay (LPA)}

Two weeks after final immunization, the spleens were taken from immunized mice, dissected and resuspended in sterile cold PBS containing 2\% FBS. the RBCs were lysed with lysis buffer, and single-cell suspension was adjusted to $2 \times 10^{6}$ cells/mL in RPMI 1640 (Gibco, Germany) supplemented with 5\% FBS, $4 \mathrm{mM}$ L-glutamine, $1 \mathrm{mM}$ sodium pyrovate, $50 \mu \mathrm{m} 2 \mathrm{ME}, 100 \mu \mathrm{g} / \mathrm{mL}$ streptomycin and 100 $\mathrm{IU} / \mathrm{mL}$ penicillin. $100 \mu \mathrm{L}$ of diluted cell suspensions were dispensed into 96-well flat-bottom culture plates and stimulated with $10 \mu \mathrm{g} / \mathrm{mL}$ of recombinant peptide as an antigen recall. Phytohemagglutinin-A (5 $\mu \mathrm{g} / \mathrm{mL}$, Gibco) was used as a positive control, un-stimulated wells were 
used as the negative controls and complete culture medium was used as blank.

All experiments were performed in triplicate. After $72 \mathrm{~h}$ of culture, $100 \mu \mathrm{L}$ of 5-bromo-2-deoxy-uridine (Brdu) labeling solution was added into each well and incubation continued for $18 \mathrm{~h}$ later. After then, the plates were centrifuged and culture medium was removed completely. The plates were dried and fixed by adding $100 \mu \mathrm{L}$ of fixation/ permeabilization buffer per well. Then, $100 \mu \mathrm{L}$ of antiBrdu antibody was added to each well, and the plates washed four times and tetramethylbenzidine solution was added to each well as the substrate in dark condition. The reaction was stopped by adding $100 \mu \mathrm{L}$ of $2 \mathrm{~N} \mathrm{H} 2 \mathrm{SO} 4$. The absorbance was measured using a spectrophotometric plate reader at $450 \mathrm{~nm}$. Stimulation Index (SI) was calculated according to the following formula: OD $450 \mathrm{~nm}$ of stimulated wells/OD $450 \mathrm{~nm}$ of un-stimulated wells (20).

\subsection{IL-4 and IFN- $\gamma$ Cytokine ELISA}

Two weeks after final immunization, a total number of 4 $\times 10^{6}$ splenocytes were plated in each well of 24 -well plate using a complete RPMI-1640 medium and stimulated in vitro with $10 \mu \mathrm{g} / \mathrm{mL}$ of r-fla-A peptide and incubated at 37 ${ }^{\circ} \mathrm{C}$ in $5 \% \mathrm{CO} 2.72 \mathrm{~h}$ after the antigen recall, supernatants were collected and centrifuged at $300 \mathrm{~g}$ for $10 \mathrm{~min}$ and stored at $-70 \circ \mathrm{C}$ for cytokine analysis. IFN- $\gamma$ and IL- 4 cytokine analysis carried out with commercial ELISA Kit (Quantikine, R\&D Systems, USA) according to the manufacturer's instruction. The quantity of each secreted cytokine reported as $\mathrm{pg} / \mathrm{mL}$ according to the standard curve (14).

\subsection{Total Antibodies ELISA}

Specific antibodies were determined by an optimized indirect ELISA method. $100 \mu \mathrm{L}$ of $10 \mu \mathrm{g} / \mathrm{mL}$ r-fla-A peptide in PBS buffer were added into 96-well ELISA plates (Greiner, Germany) and incubated over night at $4{ }^{\circ} \mathrm{C}$. The wells washed with PBS containing 0.05\% Tween 20 (washing buffer) and blocked for $2 \mathrm{~h}$ at $37{ }^{\circ} \mathrm{C}$ with $5 \%$ non-fat dry milk in PBS (blocking buffer). Plates were washed and 100 $\mu \mathrm{L}$ of 1:100 diluted sera were added into each well and incubated at $37{ }^{\circ} \mathrm{C}$ for $2 \mathrm{~h}$. The wells washed three times and incubated for $2 \mathrm{~h}$ with $100 \mu \mathrm{L}$ of 1:7000 dilution of HRPconjugated anti-mouse antibody (sigma). After five times washing, the well incubated for 30 min with $100 \mu \mathrm{L}$ of TMB substrate in the dark, and reaction was stopped with $2 \mathrm{~N} \mathrm{H} 2 \mathrm{SO} 4$ and color density was measured at $450 \mathrm{~nm}$ with the ELISA plate reader (Multiskan Lab systems) (14).

\subsection{ELISA of Specific IgG Isotypes and IgM}

Detection of specific IgG1, IgG2a, IgG2b, IgG3 and IgM subclasses were performed using indirect ELISA. after antigen coating and blocking, plates were washed and then $100 \mu \mathrm{L}$ of 1:100 diluted sera were added into each well and incubated. The wells washed three times and $100 \mu \mathrm{L}$ of 1:1000 dilution of anti-mouse IgG1, IgG2a, IgG2b, IgG3 and IgM subclasses as secondary antibodies were added. After washing three times, $100 \mu \mathrm{L}$ of HRP-conjugated anti-goat antibody (sigma) was added to each well. The plates were washed five times and incubated for 30 min with $100 \mu \mathrm{L}$ of TMB substrate in the dark. The Peroxidase-TMB reaction is stopped by addition of $2 \mathrm{~N} \mathrm{H} 2 \mathrm{SO} 4$, and the color density was measured at $450 \mathrm{~nm}$ using the ELISA plate reader (14).

\subsection{Statistical Analysis}

All experiments performed in triplicate, and the data expressed as means \pm S.D of each experiment. All statistical analyses carried out by Mann-Whitney U test. In all of the cases, P values $<0.05$ were considered to be statistically significant.

\section{Results}

\subsection{Lymphocyte Proliferation}

Result of lymphocyte proliferation of experimental groups showed that FliC could stimulate lymphocyte proliferation. As shown in Figure 1, immunization of mice by adjuvanted r-fla-A protein significantly increased lymphocyte proliferation in comparison with control groups $(\mathrm{P}=0.004)$.

Figure 1. Lymphocyte Proliferation Responses of Experimental Groups

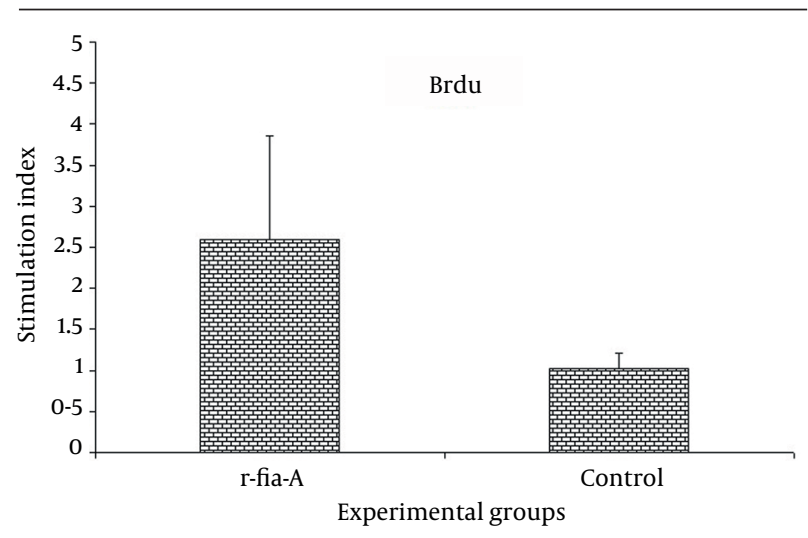

Mice ( $n=6$ mice per group) were immunized with candidate vaccine or $\mathrm{PBS}$ as the control group. Two weeks after final immunization, proliferation activity was measured with Brdu/ELISA. Results showed that immunization of mice with r-fla-A significantly induced proliferation of splenic lymphocytes compared with control group. Experiment was carried out triplicate and values are shown as the mean \pm S.D.

\subsection{ELISA of IL-4 and IFN- $\gamma$}

To detect the cytokine secretion pattern, splenocytes were cultured as described above and re-stimulated in vitro by r-fla-A peptide. Collected supernatants were monitored for the presence of IFN- $\gamma$ and IL-4 to determine the type (T helper 1 vs. T helper 2 ) of immune responses. As 
shown in Figure 2, the results showed that immunization of mice with r-fla-A induced high level of IFN- $\gamma$ (Figure $2 \mathrm{a}$ ) and IL-4 (Figure $2 \mathrm{~b}$ ) in comparison to the control group $(\mathrm{P}=0.003$ and $\mathrm{P}=0.006$, respectively). Overall, our result demonstrated that immunization of mice with $\mathrm{r}$ fla-A induced both IL-4 and IFN- $\gamma$ cytokines, but IFN- $\gamma$ was dominant. These results indicated that both arms of the immune system have been triggered.

Figure 2. IFN- $\gamma$ and IL-4 Cytokines Analysis of Experimental Groups with Quantitative ELISA After Immunization

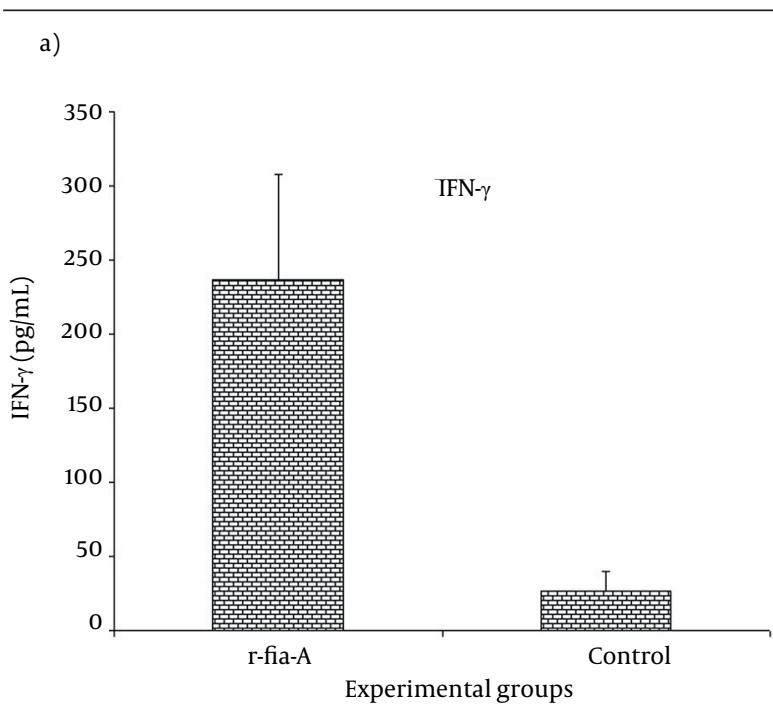

b)

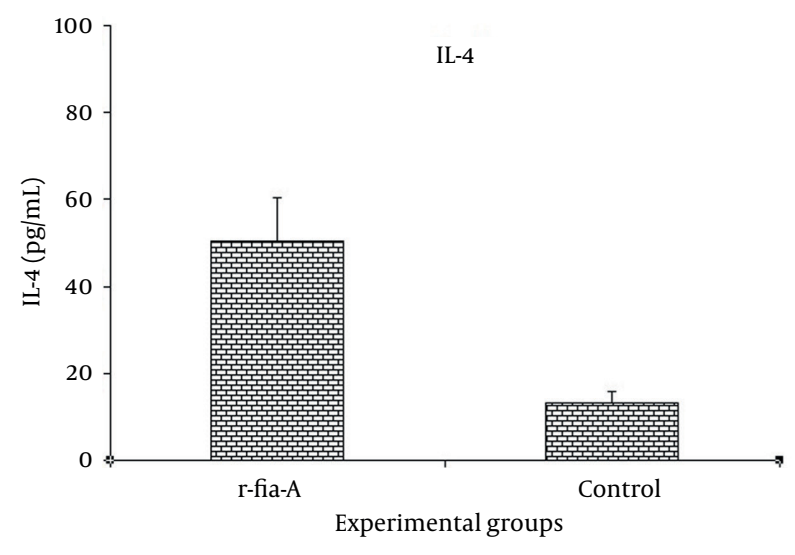

Mice were immunized with candidate vaccine, mice were injected with PBS ( $n=6$ mice per group) as control group. Results showed that immunization with r-fla-A significantly induced both IFN- $\gamma$ and IL-4 secretion compared with control group, however IFN- $\gamma$ level was dominant. Experiments carried out triplicate and values are shown as the mean \pm S.D.

\subsection{Humoral Immune Response Assay}

Specific humoral immune response was monitored with an optimized indirect ELISA. The result of the total antibody assay (Figure 3) showed that immunization of mice with r-fla-A protein induced high level of antibody response that was statistically significant with the control group $(\mathrm{P}=0.003)$.

Figure 3. Humoral Immune Responses Against Candidate Vaccine After Immunization with r-fla-A or PBS

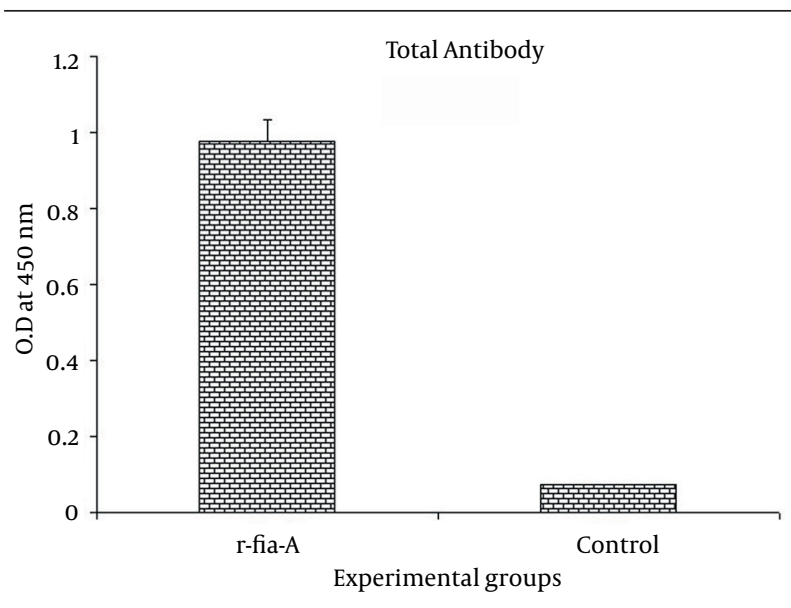

Sera of individual mice collected and antigen specific total IgG was evaluated with an optimized indirect ELISA as mentioned in materials and methods section.

\subsection{Specific Isotypes Monitoring}

To determine the specific antibody isotypes, ELISA method was used. As shown in the Figure 4, immunization of mice with r-fla-A induces specific IgG1 (Figure 4 a), IgG2a (Figure 4 b), IgG2b (Figure $4 \mathrm{c}$ ) and IgG3 (Figure $4 \mathrm{~d})$ antibody that indicate a significant difference with the control group $(\mathrm{P}=0.003, \mathrm{P}=0.004, \mathrm{P}=0.004$ and $\mathrm{P}$ $=0.006$ respectively). Furthermore, evaluation of specific IgM (Figure $4 \mathrm{e}$ ) in the experimental groups showed that immunization of mice with r-fla-A induces higher level of specific IgM compared with control group $(\mathrm{P}=0.004)$. Overall, it is seemed that immunization of mice with $\mathrm{r}$ fla-A peptide induces poly-isotypic humoral immune response. 
Figure 4. Specific Isotypes Were Detected with ELISA Using Anti IgG1, IgG2a, IgG2b, IgG3 and IgM Antibodies
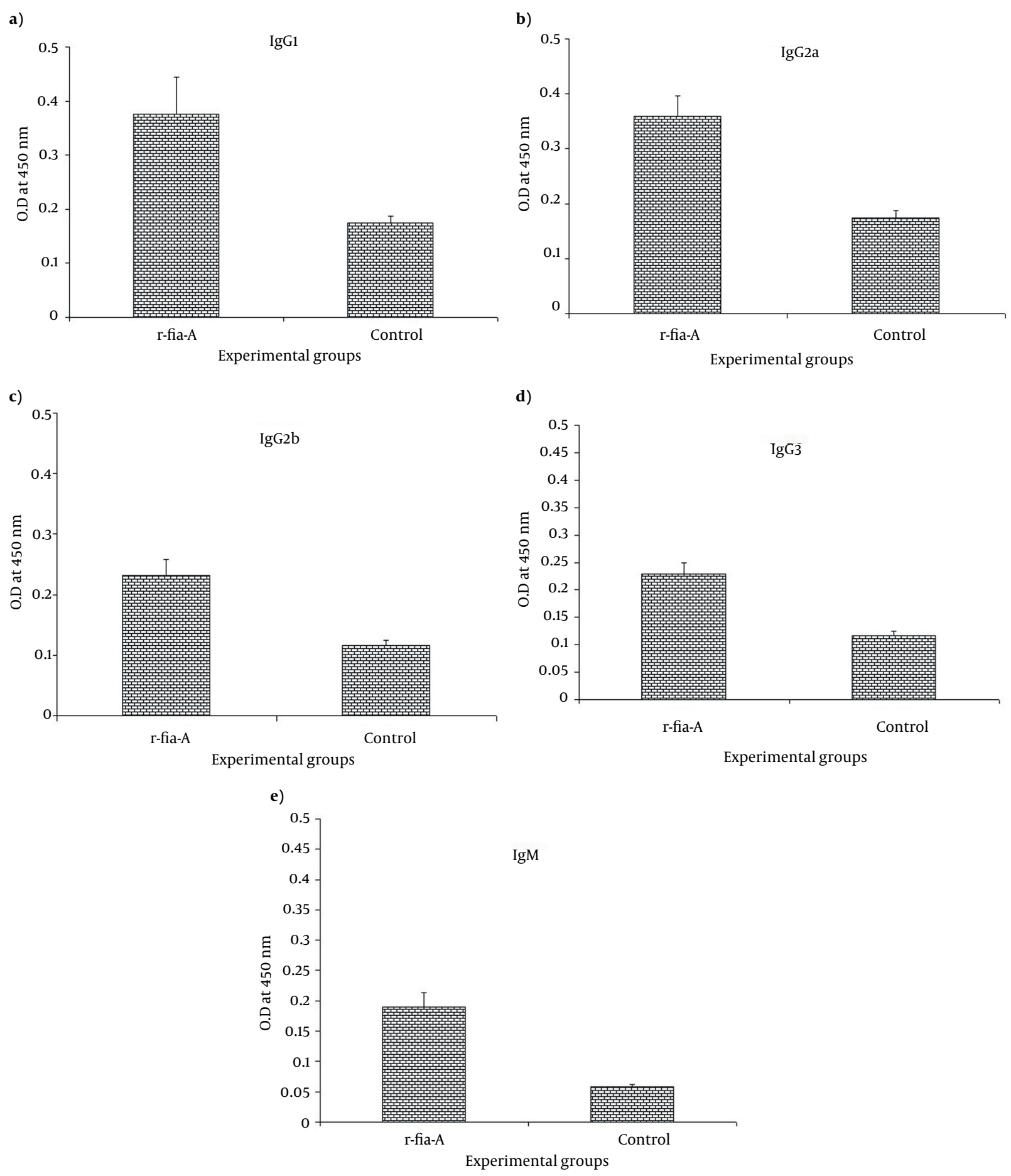

Immunization of mice with $\mathrm{r}$-fla-A significantly induced total IgG compared with control group. Also, Immunization with candidate vaccine significantly increased specific IgG1 (Figure 4a), IgG2a (Figure 4b), IgG2b (Figure 4c), IgG3 (Figure 4d) and IgM (Figure 4e) antibodies compared with control group. Experiments carried out triplicate and values are shown as the mean \pm S.D.

\section{Discussion}

There has long been recognized that flagella is one of the most effective immunologic stimulator of the immune system. The use of flagellin as a vaccine candidate, part of vaccine or adjuvant has been widely considered. 
Flagellin is a protein with intrinsic activity mediated through TLR5 therefore, can be genetically manipulated in various ways for research purpose (21-23). In other words, advances in the field of innate immunity have revealed the effective cellular and molecular mechanisms involved in pathogen-associated molecular patterns (14).

In this study, results show that r-fla-A could induce lymphocyte proliferation, considering that proliferation is a criterion of cell-mediated immunity, thus r-fla-A could promote the cellular immune response. Arnon et al. demonstrated that recombinant flagellin containing foreign epitopes caused protective immune responses in the absence of any additional adjuvant, flagellin has effects on T cells proliferation and cytokine responses $(24,25)$. The induction of potent cell-mediated immunity will be necessary and may require the induction of cytotoxic $\mathrm{T}$ cell (CTL) which kills host cells infected with intracellular pathogens (12). In addition, the innate immune system directs the balance of humoral and cellular responses, and adjuvant can control the type of acquired immune responses (26).

Results of the cytokine assay showed that r-fla-A could induce both IL- 4 and IFN- $\gamma$ secretion, but with IFN- $\gamma$ dominancy and Th1 confirmed pattern. Johansen et al. demonstrated that IFN- $\gamma$ therapy of chronic P. aeruginosa infected rats was shown to decrease the inflammatory responses and reduce the lung inflammation incidence from an acute type dominated by neutrophils to an inflammation dominated by macrophage cells (10). Studies on an acute animal infection model, have shown that immunization with killed $P$. aeruginosa induces the protective cell-mediated immunity in the lung. It has been proposed that protective responses in the lung are induced by $\mathrm{T}$ cell, followed by activated alveolar macrophages (27).

This study also showed that r-fla-A could induce specific antibodies and in this function, it induced all isotypes of specific antibodies in the mouse model. Considering this fact that each isotype of antibodies has unique function in inducing the humoral immune response, the production of poly-isotypic humoral immune response show that FliC could stimulate humoral immune response with excellent bioactivity and this argument is proved in passive and active immunotherapy of $P$. aeruginosa infections $(13,17)$. In our previous study, we proved that immunization with anti r-fla-A IgG by increasing the phagocytosis and bacterial immobilization could protect burned mice against lethal P. aeruginosa infections (75\% protection compared with $16.6 \%$ in the control IgG) (17).

The results presented in our last study, demonstrated that $r$-fla-A is a potent adjuvant to develop the humoral immune responses. Furthermore, we have shown that immunization of mice with r-fla-A could remarkably increase survival of mice infected with homologous strain (PAK) in the burn wound infection, but slight protection was observed in mice infected with heterologous strain PAO1 (unpublished result). Antibody is an important factor to control the pseudomonas infection in the active or passive form of vaccination (28). As the results of our study have shown, r-fla-A induced poly-isotypic humoral immune responses and considering that each antibody isotype may have a distinct function in the immune responses, this confirmed that r-fla-A is a strong inducer of humoral immune responses in which in the level and bioactivity of products are higher.

The poly-isotypic humoral immune response, due to its nature, could be successful in control of infection and the results of our previous study proved this competence and specific antibodies against r-fla-A could control the pseudomonas infection in the passive (17) and active (unpublished result) form of vaccination. To conclude all thee findings, our results illustrate that r-fla-A could induce cellular and humoral immune responses in murine models. The results of our study confirmed that r-fla-A could be used as a candidate vaccine or as an adjuvant in the vaccination strategy of other pathogens so, presently studies are also being conducted in our laboratories to further studies on r-fla-A as adjuvant in combination with a candidate vaccine of human papilloma virus.

\section{Acknowledgements}

This work was supported by grant No. 559 from Pasteur Institute of Iran, Tehran, Iran. The authors also thank Dr. Morteza Sattari, who passed away before the end of this project, for his collaboration in this study. .

\section{Authors' Contribution}

None declared.

\section{Financial Disclosure}

The authors of this research article have no financial conflict of interest.

\section{Funding/Support}

This work was supported by grant No. 559 from Pasteur Institute of Iran, Tehran, Iran.

\section{References}

1. Pier G. Application of vaccine technology to prevention of Pseudomonas aeruginosa infections. Expert Rev Vaccines. 2005;4(5):645-56.

2. Erol S, Altoparlak U, Akcay MN, Celebi F, Parlak M. Changes of microbial flora and wound colonization in burned patients. Burns. 2004;30(4):357-61.

3. Ramos HC, Rumbo M, Sirard JC. Bacterial flagellins: mediators of pathogenicity and host immune responses in mucosa. Trends Microbiol. 2004;12(11):509-17.

4. Winstanley C, Coulson MA, Wepner B, Morgan JA, Hart CA. Flagellin gene and protein variation amongst clinical isolates of Pseudomonas aeruginosa. Microbiology. 1996;142 ( Pt 8):2145-51.

5. McSorley SJ, Ehst BD, Yu Y, Gewirtz AT. Bacterial flagellin is an effective adjuvant for CD4+T cells in vivo.J Immunol. 2002;169(7):39149.

6. Sanders CJ, Yu Y, Moore DA, 3rd, Williams IR, Gewirtz AT. Humoral immune response to flagellin requires $T$ cells and activation of innate immunity. J Immunol. 2006;177(5):2810-8.

7. Higgins SC, Mills KH. TLR, NLR Agonists, and Other Immune 
Modulators as Infectious Disease Vaccine Adjuvants. Curr Infect Dis Rep. 2010;12(1):4-12.

8. Munster AndrewM, Leary AnneG. Cell-mediated immune responses to pseudomonas aeruginosa. Am J Surg. 1977;133(6):710-2.

9. Cripps AW, Dunkley ML, Clancy RL, Kyd J. Pulmonary immunity to Pseudomonas aeruginosa. Immunol Cell Biol. 1995;73(5):418-24.

10. Johansen HK, Hougen HP, Rygaard J, Hoiby N. Interferon-gamma (IFN-gamma) treatment decreases the inflammatory response in chronic Pseudomonas aeruginosa pneumonia in rats. Clin Exp Immunol.1996;103(2):212-8.

11. Honko AN, Sriranganathan N, Lees CJ, Mizel SB. Flagellin is an effective adjuvant for immunization against lethal respiratory challenge with Yersinia pestis. Infect Immun. 2006;74(2):1113-20.

12. Singh M, O'Hagan DT. Recent advances in vaccine adjuvants. Pharm Res. 2002;19(6):715-28.

13. Mahdavi M, Ebtekar M, Khorram Khorshid HR, Azadmanesh K, Hartoonian C, Hassan ZM. ELISPOT analysis of a new CTL based DNA vaccine for HIV-1 using GM-CSF in DNA prime/peptide boost strategy: GM-CSF induced long-lived memory responses. Immunol Lett. 2011;140(1-2):14-20.

14. Campodonico VL, Llosa NJ, Grout M, Doring G, Maira-Litran T, Pier GB. Evaluation of flagella and flagellin of Pseudomonas aeruginosa as vaccines. Infect Immun. 2010;78(2):746-55.

15. Doring G, Pier GB. Vaccines and immunotherapy against Pseudomonas aeruginosa. Vaccine. 2008;26(8):1011-24.

16. Goudarzi G, Sattari M, Roudkenar MH, Montajabi-Niyat M, Zavaran-Hosseini A, Mosavi-Hosseini K. Cloning, expression, purification, and characterization of recombinant flagellin isolated from Pseudomonas aeruginosa. Biotechnol Lett. 2009;31(9):135360.

17. Faezi S, Sattari M, Mahdavi M, Roudkenar MH. Passive immunisation against Pseudomonas aeruginosa recombinant flagellin in an experimental model of burn wound sepsis. Burns. 2011;37(5):865-72.

18. Sadikot RT, Blackwell TS, Christman JW, Prince AS. Pathogenhost interactions in Pseudomonas aeruginosa pneumonia. Am J Respir Crit Care Med. 2005;171(11):1209-23.
19. Jang SI, Lillehoj HS, Lee SH, Lee KW, Park MS, Bauchan GR, et al. Immunoenhancing effects of Montanide ISA oil-based adjuvants on recombinant coccidia antigen vaccination against Eimeria acervulina infection. Vet Parasitol. 2010;172(3-4):221-8.

20. Kyd JM, Dunkley ML, Cripps AW. Enhanced respiratory clearance of nontypeable Haemophilus influenzae following mucosal immunization with P6 in a rat model. Infect Immun. 1995;63(8):293140.

21. Saha S, Takeshita F, Matsuda T, Jounai N, Kobiyama K, Matsumoto T, et al. Blocking of the TLR5 activation domain hampers protective potential of flagellin DNA vaccine. J Immunol. 2007;179(2):1147-54.

22. Jiao XD, Hu YH, Sun L. Dissection and localization of the immunostimulating domain of Edwardsiella tarda FliC. Vaccine. 2010;28(34):5635-40.

23. Smith KD, Andersen-Nissen E, Hayashi F, Strobe K, Bergman MA, Barrett SL, et al. Toll-like receptor 5 recognizes a conserved site on flagellin required for protofilament formation and bacterial motility. Nat Immunol. 2003;4(12):1247-53.

24. Ben-Yedidia T, Marcus H, Reisner Y, Arnon R. Intranasal administration of peptide vaccine protects human/mouse radiation chimera from influenza infection. Int Immunol.1999;11(7):1043-51.

25. Honko AN, Mizel SB. Mucosal administration of flagellin induces innate immunity in the mouse lung. Infect Immun. 2004;72(11):6676-9.

26. Verma A, Arora SK, Kuravi SK, Ramphal R. Roles of specific amino acids in the $\mathrm{N}$ terminus of Pseudomonas aeruginosa flagellin and of flagellin glycosylation in the innate immune response. Infect Immun . 2005;73(12):8237-46.

27. Buret A, Dunkley ML, Pang G, Clancy RL, Cripps AW. Pulmonary immunity to Pseudomonas aeruginosa in intestinally immunized rats roles of alveolar macrophages, tumor necrosis factor alpha, and interleukin-1 alpha. Infect Immun .1994;62(12):5335-43.

28. Vassilieva EV, Wang BZ, Vzorov AN, Wang L, Wang YC, Bozja J, et al. Enhanced mucosal immune responses to HIV virus-like particles containing a membrane-anchored adjuvant. MBio. 2011;2(1):e0 0328-10. 